\title{
Continuous peripheral perfusion index in patients admitted to hospital wards - an observational study
}

Rasmussen, P. S.; Aasvang, E. K.; Olsen, Rasmus Munch; Haahr-Raunkjaer, C.; Elvekjaer, M.; Sørensen, Helge Bjarup Dissing; Meyhoff, C. S.

Published in:

Acta Anaesthesiologica Scandinavica

Link to article, DOI:

10.1111/aas.13711

Publication date:

2021

Document Version

Peer reviewed version

Link back to DTU Orbit

Citation (APA):

Rasmussen, P. S., Aasvang, E. K., Olsen, R. M., Haahr-Raunkjaer, C., Elvekjaer, M., Sørensen, H. B. D., \& Meyhoff, C.S. (2021). Continuous peripheral perfusion index in patients admitted to hospital wards - an observational study. Acta Anaesthesiologica Scandinavica, 65(2), 257-265. https://doi.org/10.1111/aas.13711

\section{General rights}

Copyright and moral rights for the publications made accessible in the public portal are retained by the authors and/or other copyright owners and it is a condition of accessing publications that users recognise and abide by the legal requirements associated with these rights.

- Users may download and print one copy of any publication from the public portal for the purpose of private study or research.

- You may not further distribute the material or use it for any profit-making activity or commercial gain

- You may freely distribute the URL identifying the publication in the public portal 
Title: Continuous peripheral perfusion index in patients admitted to hospital wards - an observational study

Rasmussen PS. ${ }^{1,2,3}$, Aasvang EK..$^{3,5^{*}}$, Olsen RM. ${ }^{4}$, Haahr-Raunkjaer C. ${ }^{1,2,3}$, Elvekjaer M. ${ }^{1,2,3}$, Sørensen HBD. ${ }^{4 *}$, Meyhoff CS. ${ }^{1,2,5^{*}}$.

${ }^{1}$ Department of Anaesthesia and Intensive Care, Bispebjerg and Frederiksberg Hospital, University of Copenhagen, Copenhagen, Denmark

${ }^{2}$ Copenhagen Center for Translational Research, Copenhagen University Hospital, Bispebjerg and Frederiksberg, Copenhagen, Denmark

${ }^{3}$ Department of Anaesthesiology, Centre for Cancer and Organ Diseases, Rigshospitalet, University of Copenhagen, Copenhagen, Denmark

${ }^{4}$ Biomedical Engineering, Department of Health Technology, Technical University of Denmark, Kgs. Lyngby, Denmark

${ }^{5}$ Department of Clinical Medicine, University of Copenhagen, Copenhagen, Denmark

*Aasvang EK., Sørensen HBD. and Meyhoff CS. shared last authorship.

\section{Abstract count: 250}

Word count: 3000

\section{Short title: Continuous peripheral perfusion index}

\section{Corresponding author:}

Christian Sylvest Meyhoff, MD, Ph.D.

Department of Anaesthesia and Intensive Care, Bispebjerg and Frederiksberg Hospital, University of Copenhagen, Bispebjerg Bakke 23, DK-2400, Copenhagen, Denmark

Phone: (+45) 24910542

Fax: (+45) 38639859

E-mail address: christian.sylvest.meyhoff@regionh.dk

Conflict of interest: Elvekjaer M. reports departmental research funding from Merck Sharp \& Dome Corp. outside the submitted work. Meyhoff CS. reports direct and indirect research funding from Ferring Pharmaceuticals, Merck Sharp \& Dohme Corp. and Boehringer Ingelheim outside

This article has been accepted for publication and undergone full peer review but has not been through the copyediting, typesetting, pagination and proofreading process, which may lead to differences between this version and the Version of Record. Please cite this article as doi: 10.1111/AAS.13711

This article is protected by copyright. All rights reserved 
the submitted work. Other Authors report none. Meyhoff CS. and Aasvang EK. report speaker fee from Radiometer.

\section{Abstract}

Introduction:

Risk patients admitted to hospital wards may quickly develop heamodynamic deterioration and early recognition has high priority to allow preventive intervention. The peripheral perfusion index (PPI) may be an indicator of circulatory distress by assessing peripheral perfusion non-invasively from photoplethysmography. We aimed to describe the characteristics of PPI in hospitalised patients since this is not well studied.

Materials and methods:

Patients admitted due to either acute exacerbation of chronic obstructive pulmonary disease (AECOPD) or after major abdominal cancer surgery were included in this study. Patients were monitored continuously up to 96 hours with a pulse oximeter. Comparisons between median PPI each day, time of day and admission type were described with mean difference (MD) and analysed using Wilcoxon rank sum test and related to morbidity and mortality.

Results:

PPI data from 291 patients were recorded for a total of 9,279 hours. Median PPI fell from 1.4 (inter quartile range, IQR 0.9-2.3) on day 1 to 1.0 (IQR 0.6-1.6) on day 4. Significant differences occurred between PPI day vs. evening ( $\mathrm{MD}=0.18,95 \% \mathrm{CI} 0.16-0.20, \mathrm{P}=0.028$ ), day vs. night $(\mathrm{MD}=0.56,95 \%$ CI $0.49-0.62, \mathrm{p}<0.0001)$ and evening vs. night $(\mathrm{MD}=0.38,95 \% \mathrm{CI} 0.33-0.42$, $\mathrm{p}=0.002$ ). No significant difference in median PPI between AECOPD and surgical patients was found $(\mathrm{MD}=0.15,95 \% \mathrm{CI}-0.08-0.38, \mathrm{p}=0.62)$.

Conclusion:

Lower PPI during daytime vs. evening and night-time were seen for both populations. The highest frequency of serious adverse events and mortality was seen among patients with low median PPI. The clinical impact of PPI monitoring needs further confirmation.

Editorial Comment:

This article is protected by copyright. All rights reserved 
Light-based plethysmographic signals and the peripheral pulse index derived from these may be affected when there is general circulatory deterioration. This observatiopnal study explored the peripheral pulse index in 2 hospital cohorts where some circulatory compromise may be expected. Patterns in this index are described over time, by day vs night, and where very low signals may be markers of coming complications.

This article is protected by copyright. All rights reserved 


\section{Introduction}

A common risk for comorbid patient groups is in-hospital haemodynamic deterioration, ${ }^{1,2}$ where early recognition and treatment may be crucial in order to prevent further deterioration. Examples are chronic obstructive pulmonary disease (COPD) patients who have a high prevalence of comorbidities, ${ }^{3}$ and surgical patients who have increased risk of multiple complications as a result of increased inflammation ${ }^{4}$ or direct surgical complications such as bleeding, infection and anastomotic leakage. ${ }^{5,6}$ Abnormal vital signs are potential predictors of complications and mortality, thus, risk prediction and triage scoring systems, such as the early warning score (EWS), have been implemented as part of the clinical assessment. ${ }^{78}$ However, its usefulness is limited by the diversity of patient diseases and the intermittent scoring of patients. Continuous automatic vital sign monitoring could surpass the inadequacy of intermittent manual scoring of patients if implemented in hospital wards. ${ }^{9}$

A potential simple and clinically available method for evaluation of the peripheral perfusion is the peripheral perfusion index (PPI). PPI is a non-invasive clinical assessment of the microcirculation based on photoelectric plethysmographic measurement. It measures the ratio between the pulsatile and non-pulsatile blood flow in the peripheral tissues (e.g. fingertip, toe or ear lobe) with a pulseoximeter. ${ }^{10,11}$ Therefore, it is a relative assessment of pulse strength in the peripheral circulation not taking the patient's blood oxygen saturation into consideration. ${ }^{12}$ The peripheral tissue perfusion is often the first region to be compromised during haemodynamic deterioration of patients, when global oxygen demand exceeds global oxygen delivery as traditionally seen in circulatory shock. ${ }^{13}$ During shock conditions the peripheral tissue bed is constricted to increase the central blood volume and perfusion of the vital organs. ${ }^{14}$ This decreases the pulsatile arterial amplitude and thus lowers the PPI. ${ }^{15}$ In contrast, vasodilatation from sepsis, septic shock or increased cardiac output would increase the PPI. ${ }^{16}$ A number of other factors have the potential to affect PPI to similar extent such as pain, hypovolemia, patient positioning, fever and environmental temperature. ${ }^{17-19}$ Continuously monitored PPI can be used perioperatively and in the intensive care setting as a marker of vasoconstriction or -dilatation and potentially to guide evaluation of cardiac output and clinical interventions to improve perfusion..$^{15,20,21}$

However, neither the characteristics nor variation of PPI throughout the day have been fully investigated in the haemodynamic assessment of patients admitted to hospital wards. The aim of 
this observational study was to describe characteristics of the PPI, when measured continuously in patients hospitalised due to either acute exacerbation of COPD (AECOPD) or after major abdominal cancer surgery with emphasis on changes over days and time of day. Furthermore, associations between median PPI and serious adverse events and mortality were analysed. 


\section{Methods}

This study collected data from two ongoing trials (NCT03660501 and NCT03491137), which were approved by the Danish Data Protection Agency and regional ethics committee (Protocol number 18026653 and 17033535). All participating patients gave informed consent before inclusion.

Setting and patient population

Inclusion criteria were admission to the hospital due to AECOPD (age $\geq 18$ years) or elective major abdominal cancer surgery with an estimated operation time of $\geq 2$ hours (age $\geq 60$ years). The patients were excluded if they were unable to cooperate or give informed consent, had a pacemaker/ICD, had severe allergy for band aid, plastic or silicone, or withheld active treatment. Additionally, the surgical patients had to pass a mini-mental state examination (score of $\geq 24$ ).

The surgical patients were screened and included prior to their operation and monitoring was initiated in the hours following surgery or upon post-anaesthesia care unit (PACU) discharge to the general ward. Monitoring was initiated on average 11 hours after arrival to PACU. The AECOPD patients were included with monitoring initiated within $24 \mathrm{~h}$ from admission to the hospital.

\section{Data sources}

As part of the WARD-monitoring set-up, ${ }^{1}$ pulse oximeter data were collected using a wrist-borne pulse oximeter (Nonin WristOx 3150, Nonin Medical Inc, Minnesota, USA) with the sensor on a random finger of choice. Besides measuring the peripheral oxygen saturation $\left(\mathrm{SpO}_{2}\right)$, the PPI was extracted from the photoplethysmographic waveform. The study did not affect standard care as data from the pulse oximeter were wirelessly transferred via Bluetooth to a bedside gateway, blinded from clinical staff. Participating patients were visited at least once a day by the investigators to change batteries and to encourage compliance to the study. The device had to be worn continuously for up to 96 hours after inclusion or until discharge from the hospital, which corresponded to the battery time of the heart- and respiratory rate monitor in the WARD system. However, the patients were instructed to take off the device prior to bathing or diagnostic imaging. Follow-up was performed at 30 days for clinical outcomes, which was retrieved from the electronic medical record of all patients. All non-fatal serious adverse events (SAE) and mortality 
(according to the International Conference on Harmonisation guidelines for Good Clinical Practice) was categorised according to a manual with instructions on how to report each relevant adverse events and all serious adverse events according to international definitions.

Data analysis

The plethysmographic waveform from pulse oximetry illustrates a pulsatile arterial component (AC) and a non-pulsatile component (DC), which arise from the absorption of infrared light. ${ }^{11}$ The PPI was calculated as the ratio between AC and DC.

Some PPI ratios were too high to calculate with exact number, since a PPI of unknown exact magnitude would occur in cases, where the AC was outside of range with a low DC. As such, a very high PPI defined as ratios of 10 or higher were used in our analyses as the value 10. Such high calculations of PPI $\geq 10$ occurred in $2 \%$ of total PPI monitoring time. Invalid values due to signal noise (most often caused by motion artefacts) as well as other artefacts were manually and automatically removed from the raw data according to the Signal Quality Index (SQI). ${ }^{22}$ Data were collected with a sampling frequency of $1 \mathrm{~Hz}$ and were presented as median PPI-values, which required at least five continuous reliable measurements by the pulse oximeter per minute to calculate. Data were presented as a 10-minute average based on these values due to fluctuations. Missing data, due to invalid recording or removal of the pulse oximeter, ranging from minutes to hours were replaced by mean values based on valid data before and after the missing period.

In the primary analysis the median PPI data were divided into four different groups defined according to earlier research: ${ }^{10,23}$ Low median PPI $(\leq 0.6)$, mild impaired median PPI $(0.6-1.4)$, normal median PPI (1.4 - 5.0) and high median PPI ( $\geq 5.0)$. Data were presented as median PPI on day 1 , day 2, day 3 and day 4. Furthermore, according to daytime (08:00-16:00), evening (16:0000:00) and night-time (00:00-08:00) with intergroups comparison of median values measured in the respective time intervals. We added a post hoc explorative description of the frequency of any SAE and mortality within 30 days according to median PPI. Also, we included a post hoc analysis of overall median PPI and median PPI in daytime, evening and night-time on data without interpolated values.

Statistical analysis

This article is protected by copyright. All rights reserved 
Data were presented as medians with interquartile range and mean differences between groups with 95\% confidence interval. For the statistical analysis, SAS version 9.4 (SAS institute, Cary, NC, USA) was used. Comparisons between the AECOPD and surgical groups were performed using the Wilcoxon rank sum test, and comparison between time of day was performed using the paired t-test. We calculated univariate odds ratios (OR) for the risk of SAE according to PPI group with logistic regression. All with $\mathrm{P}<.05$ considered statistically significant. 


\section{Results}

Patients were included from February 2018 to March 2019 at Bispebjerg Hospital and Rigshospitalet, Denmark. In the AECOPD and surgical substudies, 88 and 203 patients were enrolled, respectively. The AECOPD patients were only admitted to Bispebjerg Hospital, while surgical patients were admitted to both Bispebjerg Hospital and Rigshospitalet. Baseline characteristics are described in table 1.

The 291 patients were monitored for an overall observation time of 18,510 hours averaging 64 (IQR 43-94) hours per patient (figure 1). Recorded Data used for PPI calculations were a total of 9,279 hours averaging 32 (IQR 15-46) hours per patient. The recorded data accounted for $50 \%$ of the total observation time with a distribution of $44 \%$ and $53 \%$ for the AECOPD and surgical group, respectively. PPI were recorded in $43 \%$ of daytime, $51 \%$ of evening and $56 \%$ of night-time, and measurement time were 2,799, 2,784, 2,031 and 1,665 hours on day 1, 2, 3 and 4, corresponding to the number of patients still admitted (figure 1).

The overall median PPI among all patients was 1.3 both with (IQR 0.8-2.0) or without (0-8-2.1) interpolation. We found no difference in the overall median PPI between the AECOPD and surgical group $(\mathrm{MD}=0.15,95 \% \mathrm{CI}-0.08-0.38, \mathrm{p}=0.67$, figure 2). Most patients' PPI were in the interval of 0.6-1.4 (44\%) and 1.4-5.0 (39\%). Fifty-six percent of the patients had a PPI under 1.4 in more than $50 \%$ of the total measured time and $12 \%$ had a PPI under 0.6 in more than half of the monitoring period. The average time for each patient in the same PPI category was $68 \%$.

A total of 117 patients (40\%) developed one or more SAE within 30 days, and 30-day mortality was $3,4 \%(n=10)$. In the four groups of median PPI, SAE occurred in $62 \%, 42 \%, 34 \%$ and $21 \%$ for median PPI <0.6, 0.6-1.4, 1.4-5.0 and >5.0 (p-values: 0.004, 0.22, REF and 0.34, respectively). OR for developing one or more SAE in PPI groups <0.6, 0.6-1.4 and $>5.0$ were 3.11 (95\% CI 1.41-6.86), 1.38 (0.82-2.33) and 0.52 (0.14-1.99), when comparing to PPI group 1.4-5.0 (table 2), 
respectively. OR for PPI below 0.3 in more than 2.5, 5 and 10 hours were 2.51 (1.52-4.16), 3.60 (1.80-7.18) and 5.78 (1.85-18.02). Thirty-day mortality were 5.9\%, 4.7\%, 1.8.\% and 0\%, respectively (figure 2).

A median PPI of 1.4 (IQR 0.9-2.3) was found on day 1 ( $\mathrm{n}=282)$; median PPI of 1.2 (IQR 0.8-2.1) on day $2(\mathrm{n}=261)$; median PPI of 1.1 (IQR 0.6-1.7) on day $3(\mathrm{n}=226)$; median PPI of 1.0 (IQR 0.61.6) on day $4(\mathrm{n}=166)$. Median PPI on day 1 , both daytime, evening and night-time, was higher compared to median PPI on day 4 (figure $3 a$ and $3 b$ ).

The AECOPD patients had an overall median PPI of 1.2 (IQR 0.8-2.0), while being 1.1 (IQR 0.71.9), 1.3 (IQR 0.8-2.0) and 1.2 (IQR 0.8-2.3) for day, evening and night, respectively. The overall median PPI for surgical patients was 1.3 (IQR 0.8-2.0), while being 1.0 (IQR 0.7-1.7), 1.2 (IQR 0.7-1.9) and 1.6 (IQR 0.9-2.4) for day, evening and night, respectively.

Daytime median PPI was 1.0 (IQR 0.7-1.7), evening median PPI was 1.2 (IQR 0.7-1.9) and nighttime median PPI was 1.5 (IQR 0.9-2.3) (figure 4). Without interpolation, daytime, evening and night-time median PPI were 1.1 (0-7-1.8), 1.3 (0.8-2.1) and 1.5 (1.0-2.4), respectively. Comparing median PPI with interpolation, we found significant differences for the following comparisons: Day vs evening $(\mathrm{MD}=0.22,95 \%$ CI $0.12-0.31, \mathrm{P}<.0001)$, day vs night $(\mathrm{MD}=0.62,95 \% \mathrm{CI} 0.44-$ $0.79, \mathrm{P}<.0001)$ and evening vs night $(\mathrm{MD}=0.39,95 \% \mathrm{CI} 0.24-0.54, \mathrm{P}<.0001)$. 


\section{Discussion}

This observational study examined the peripheral perfusion index and its distribution in the first 96 hours after admission to hospital for AECOPD or during the first four days after major abdominal cancer surgery. A median PPI of 1.3 was found among all included patients with no significant difference between the AECOPD and the surgical groups. Interpolated data did not differ in median PPI from data without interpolation. The median PPI ranged from 0.1 to 10 (maximum value), where $12 \%$ of the included patients had a median PPI below 0.6 , which is defined as a low PPI. The highest frequency of SAE and mortality was seen among patients with a low median PPI. Also, exposure to a PPI below 0.3 in more than 2.5 hour was correlated to higher risks of developing one or more SAE.

The observed point estimate of median PPI appeared to be reduced from day one to day four, but this should be interpreted with caution, since there may be several explaining factors. Acute exacerbation of COPD ranges from mild to severe. Mild or moderate exacerbations are most common and stabilise within the first days, while severe exacerbations require prolonged hospitalisation. ${ }^{24}$ Enhanced recovery after surgery ${ }^{25}$ will also result in better conditions for surgical patients after major abdominal procedure shortly after surgery. ${ }^{26}$ Patients not following the intended recovery due to complications will require prolonged hospitalisation. Thus, a lower median PPI seen on day four compared to day one may represent patients with inadequate recovery or complications, because the most fit patients are already discharged.

Our study found a statistically significant difference in the PPI measured during daytime, evening and night-time. The measured PPI values were higher during evening and night hours than during daytime with mean differences of 0.18 and 0.56 , respectively. One explanation could be that patients in the evening and at night most often lie in the supine position and therefore theoretically may have increased cardiac output (due to increased preload). This reduces the systemic vascular resistance by peripheral vasodilation increasing the peripheral perfusion. Other explanations could be a calmer positioning at night, or that peripheral perfusion is influenced by whether the patients are sleeping or awake. This was reported in a study by Cresi et al. measuring the PPI on 30 preterm newborns. ${ }^{27}$ They argued, that PPI recording also could be influenced by the circadian rhythm and sleep/wake state. 
Lima et al. ${ }^{10}$ measured the PPI on 108 healthy volunteers and found a median of 1.4 (0.7-3.0), which is comparable to our results. They compared their results to critically ill patients, where a PPI of 1.4 were used to discriminate the detection of abnormal peripheral perfusion reflected by an abnormal core-to-toe temperature difference (sensitivity $81 \%$ and specificity $86 \%$ ). He et al. reported a lower discriminating PPI than Lima of 0.6 following resuscitation in predicting 30 -day mortality in critically ill patients (sensitivity $61 \%$ and specificity $90 \%$ ). ${ }^{23}$ Mildly impaired PPI (0.6-1.4) did not differ from normal PPI (>1.4) regarding outcome. Van Genderen et al. found that PPI was significantly different in patients, undergoing major abdominal surgery, who developed severe complications $(\mathrm{PPI}=1.7)$ compared to patients with non-severe complications from postoperative day two $(\mathrm{PPI}=3.9)^{28}$.

We found that more than half of our included patients had either low PPI $(<0.6,12 \%)$ or mild impaired PPI (0.6-1.4, 44\%). These two groups of patients had 46\% SAE and $4.9 \%$ mortality within 30 days as compared to $33 \%$ SAE and 1.6\% mortality among patients with normal or high median PPI. It is, however, difficult to compare a 'healthy' population in hospital wards with critically ill patients in the ICU, because the peripheral perfusion in critically ill patients often is modified by vasoactive drugs and altered self-regulation. Lima et al. also discussed this issue by suggesting that the lower limit for healthy patients probably is closer to $0.3 .{ }^{10}$ Comparing our results (median PPI of 1.3) with the studies on a normal population and in critically ill patients, it can be argued that our patient groups might fall in between these populations with normal peripheral perfusion and haemodynamic disturbance, respectively.

We based the total measurement per patient on approximately $50 \%$ of total observation time, with missing data being replaced with data from before and after the missing period. Missing data could have affected our results with reasons spanning from poor compliance to wear the device and medical issues such as delirium ${ }^{29}$ to technical difficulties such as Bluetooth connection issues or lacking battery power. Consequently, the pulse oximeter could be removed by the patient or nursing staff if the device was causing discomfort. In these situations, the PPI could be lower than the replaced mean values. Contrary, the patients could have had a higher PPI, than simplified by the mean, during periods of no recording, if the PPI only lowered in the late part of these periods. In addition, the patients did not wear the pulse oximeter during personal hygiene and diagnostic procedures. These issues are comparable with another observational study, where data collection with the pulse oximeter was achieved for $65 \%$ of the total monitoring time. ${ }^{1}$ Different 
circumstances can affect the accuracy of pulse oximeters, why measuring and calculating the PPI based on pulse oximetry has its limitations. Importantly, artefacts due to motion or low perfusion decrease the performance and precision of pulse oximeters. ${ }^{30}$ Consequently, discrepancy is seen in surgical patients, who are often confined to bed during the first days after their operations followed by movement around the wards subsequently. Since factors such as pain status or motion artefacts as well as the biological variability of low peripheral perfusion is not taken into account in this study, our results should be interpreted with caution. Accordingly, several factors must be considered when assessing the peripheral perfusion index. Considering patients in the hospital wards, several factors are, however, difficult to correct such as temperature or body positioning throughout the day. Future studies of more detailed variations of PPI are also highly relevant.

The peripheral perfusion index was measured continuously in patients admitted to general wards for up to 96 hours. This is an important initial step towards developing alarms for specific changes, which require knowing data changes and associations to outcomes. This study differs from other studies on PPI, since continuous monitoring during both daytime, evening and nighttime was considered in the overall result assessment. Our study population of hospitalised patients on general wards was also very heterogenous compared with previous studies with more standardised conditions (e.g. in the intraoperative or neonatal intensive care setting).

We conclude that similar distributions of PPI are present in patients admitted to hospital after major abdominal surgery or due to AECOPD, with a marginally lower median PPI compared to the healthy population. The highest frequency of SAE and mortality was seen among patients with low median PPI. The PPI was higher in the evening and night-time than during daytime. Time of day should be included in the overall assessment at hospital wards. Future research should confirm the clinical impact of PPI changes.

\section{Acknowledgements}

Funding:

Elvekjaer M. and Haahr-Raunkjaer C.: Received funding from Copenhagen Center for Health Technology (CACHET). The WARD-Project Group received funding from Innovation Fund Denmark, The Danish Cancer Society and The A. P. Møller Foundation. 
Other authors: Departmental funding only.

\section{References}

1. Duus CL, Aasvang EK, Olsen RM, Sørensen HBD, Jørgensen LN, Achiam MP, Meyhoff CS. Continuous vital sign monitoring after major abdominal surgery—Quantification of micro events.Acta Anaesthesiol Scand 2018; 62: 1200-8.

Elvekjaer M, Aasvang EK, Olsen RM, Sørensen HBD, Porsbjerg CM, Jensen JU, Raunkjær CH, Meyhoff CS, Group W. Physiological abnormalities in patients admitted with acute exacerbation of COPD : an observational study with continuous monitoring.J Clin Monit Comput 2019. doi: 10.1007/s10877-019-004158

3. Divo M, Cote C, De Torres JP, Casanova C, Marin JM, Pinto-Plata V, Zulueta J, Cabrera C, Zagaceta J, Hunninghake G, Celli B. Comorbidities and risk of mortality in patients with chronic obstructive pulmonary disease.Am J Respir Crit Care Med 2012; 186: 155-61.

4. Steinthorsdottir KJ, Kehlet H, Aasvang EK. Surgical stress response and the potential role of preoperative glucocorticoids on post-anesthesia care unit recovery.Minerva Anestesiol 2017; 83: 1324-31.

5. Jakobson T, Karjagin J, Vipp L, Padar M, Parik AH, Starkopf L, Kern H, Tammik O, Starkopf J. Postoperative complications and mortality after major gastrointestinal surgery.Med 2014; 50: 111-7.

Straatman J, Cuesta MA, de Lange - de Klerk ESM, van der Peet DL. Long-Term Survival After Complications Following Major Abdominal Surgery.J Gastrointest Surg 2016; 20: 1034-41.

7. Bleyer AJ, Vidya S, Russell GB, Jones CM, Sujata L, Daeihagh P, Hire D. Longitudinal analysis of one million vital signs in patients in an academic medical center.Resuscitation 2011; 82: 1387-92.

8. Goldhill, D. R., McNarry A.F., Mandersloot G. MA. A physiologically-based early warning score for ward patients: the association between score and outcome.Anaesthesia 2005; 60: 547-53.

9. Haahr-Raunkjær C, Meyhoff CS, Sørensen HBD, Olsen RM, Aasvang EK. Technological aided assessment of the acutely ill patient - The case of postoperative complications.Eur J Intern Med 2017; 45: 41-5.

10. Pinto Lima A, Beelen P, Bakker J. Use of a peripheral perfusion index derived from the pulse oximetry signal as a noninvasive indicator of perfusion.Crit Care Med 2002; 30: 1210-3.

11. Lima A, Bakker J. Noninvasive monitoring of peripheral perfusion.Intensive Care Med 2005; 31: $1316-26$. 
12. Van Genderen ME, Van Bommel J, Lima A. Monitoring peripheral perfusion in critically ill patients at the bedside.Curr Opin Crit Care 2012; 18: 273-9.

13. Vincent J-L, De Backer D. Circulatory Shock.N Engl J Med 2013; 18369: 1726-34.

14. Shah P, Cowger JA. Cardiogenic shock.Crit Care Clin 2014; 30: 391-412.

15. Corsini I, Cecchi A, Coviello C, Dani C. Perfusion index and left ventricular output correlation in healthy term infants.Eur J Pediatr 2017; 176: 1013-8.

16. Russell JA, Rush B, Boyd J. Pathophysiology of Septic Shock.Crit Care Clin 2018; 34: 43-61.

17. Høiseth L, Hisdal J, Hoff IE, Hagen OA, Landsverk SA, Kirkebøen KA. Tissue oxygen saturation and finger perfusion index in central hypovolemia: influence of pain.Crit Care Med 2015; 43: 747-56.

18. Tapar H, Karaman S, Dogru S, Karaman T, Sahin A, Tapar GG, Altiparmak F, Suren M. The effect of patient positions on perfusion index.BMC Anesthesiol 2018; 18: 1-4.

19. Yanagisawa O, Homma T, Okuwaki T, Shimao D, Takahashi H. Effects of cooling on human skin and skeletal muscle.Eur J Appl Physiol 2007; 100: 737-45.

20. Klijn E, Groeneveld ABJ, Van Genderen ME, Betjes M, Bakker J, Van Bommel J. Peripheral Perfusion Index Predicts Hypotension during Fluid Withdrawal by Continuous Veno-Venous Hemofiltration in Critically Ill Patients.Blood Purif 2015; 40: 92-8.

21. Foss NB, Kehlet H. Perioperative haemodynamics and vasoconstriction: time for reconsideration?Br J Anaesth 2019; 123: 100-3.

22. Vallance D, Bonnici T, Tarassenko L, Charlton P, Orphanidou C, Clifton D. Signal Quality Indices for the Electrocardiogram and Photoplethysmogram: Derivation and Applications to Wireless Monitoring.IEEE J Biomed Heal Informatics 2014; 19: 1-1.

23. He H, Long Y, Liu D, Wang X, Zhou X. Clinical classification of tissue perfusion based on the central venous oxygen saturation and the peripheral perfusion index.Crit Care 2015; 19: 1-10.

24. Reis AJ, Alves C, Furtado S, Ferreira J, Drummond M, Robalo-Cordeiro C. COPD exacerbations: management and hospital discharge.Pulmonology 2018; 24: 345-50.

25. Tiernan JP, Liska D. Enhanced Recovery After Surgery: Recent Developments in Colorectal Surgery.Surg Clin North Am 2018; 98: 1241-9.

26. Thiele RH, Rea KM, Turrentine FE, Friel CM, Hassinger TE, Goudreau BJ, Umapathi BA, Kron IL, Sawyer RG, Hedrick TL, McMurry TL. Standardization of care: Impact of an enhanced recovery protocol on length of stay, complications, and direct costs after colorectal surgery.J Am Coll Surg 2015; 220: 430-43.

27. Cresi F, Pelle E, Calabrese R, Costa L, Farinasso D, Silvestro L. Perfusion index variations in clinically and 
hemodynamically stable preterm newborns in the first week of life.Ital J Pediatr 2010; 36: 1-5.

28. van Genderen ME, Paauwe J, de Jonge J, van der Valk RJP, Lima A, Bakker J, van Bommel J. Clinical assessment of peripheral perfusion to predict postoperative complications after major abdominal surgery early: A prospective observational study in adults.Crit Care 2014; 18: 1-13.

29. Kwatra MM. Delirium in older persons.N Engl J Med 2006; 354: 2510.

30. Aaron Louie, B.S., John R. Feiner, M.D., Philip E. Bickler, M.D., Ph.D., Laura Rhodes BS, Michael Bernstein, B.S., Jennifer Lucero M. Four Types of Pulse Oximeters Accurately Detect Hypoxia during Low Perfusion and Motion.Anaesthesiology 2018; 128: 520-30.

\section{Legends}

Figure 1 - Trial flowchart 
Figure 2 - Distribution of overall median PPI

Distribution of median PPI based on up to 96 hours of measurement divided into intervals and 30-day outcome. AECOPD, acute exacerbation of chronic obstructive pulmonary disease. PPI, peripheral perfusion index. SAE, serious adverse event.

Figure 3 - Distribution of median PPI compared to time of day

Daytime, 08:00-16:00. Evening, 16:00-00:00. Night-time, 00:00-08:00. The boxplots are described with a median and mean value, interquartile range, whiskers and the closest outliers.

Figure 4 - Comparison between time of day

Daytime, 08:00-16:00. Evening, 16:00-00:00. Night-time, 00:00-08:00. The boxplots are described with a median and mean value, interquartile range, whiskers and the closest outliers.

Statistical differences were found in: Daytime vs. evening ( $\mathrm{MD}=0.18, \mathrm{P}=0.028)$, evening vs. night-time $(\mathrm{MD}=0.38, \mathrm{P}=0.002)$ and daytime vs. night-time $(\mathrm{MD}=0.56, \mathrm{P}<0.0001)$.

\section{Table 1 - Baseline characteristics}

Values are median [interquartile range] or number (percentage).

GOLD, the Global Initiative for Chronic Obstructive Lung Disease. FEV1/FVC, Forced Expiratory

Volume/Forced Vital Capacity. Other (location of surgery), combined surgical resections with no specific resection site.

${ }^{a}$ Danish Health Authority recommends $24 \mathrm{~g} / \mathrm{d}$ for men and $12 \mathrm{~g} / \mathrm{d}$ for women.

Table 2 - SAE and mortality

Unadjusted odds ratios (OR) for SAE according to PPI interval and exposure to PPI below 0.3. All fatalities were among patients with SAE. PPI, peripheral perfusion index. SAE, serious adverse events. CI, confidence interval. REF, reference value. 
Parameter

AECOPD Surgery

$(\mathbf{n}=\mathbf{8 8})$

$(\mathrm{n}=\mathbf{2 0 3})$

Sex (male/female), n

$44 / 44$

$112 / 91$

Age (yr)

Body mass index $\left(\mathrm{kg} / \mathrm{m}^{2}\right)$

73 [64-81]

71 [66-75]

25 [21-29]

26 [23-28]

Baseline haemodynamics

Systolic blood pressure, $\mathrm{mmHg}$

136 [119-155]

136 [123-147]

Diastolic blood pressure, $\mathbf{m m H g}$

$72[64-80]$

75 [68-81]

Pulse rate, min $^{-1}$

83 [72-93]

American Society of Anaesthesiologists class, n $(\%)$

I / II / III / IV

GOLD, $\mathbf{n}(\%)$

GOLD I

$9(10 \%)$

GOLD II

$22(25 \%)$

GOLD III

$32(36 \%)$

GOLD IV

$19(22 \%)$

Unknown

$6(7 \%)$

Smoking status, n (\%)

Current smoker

$$
\begin{gathered}
32(36 \%) \\
53(60 \%) \\
3(3 \%) \\
52[41-58] \\
18(20 \%)
\end{gathered}
$$$$
34(16 \%)
$$$$
110(54 \%)
$$$$
59(28 \%)
$$$$
72[68-78]
$$

Alcohol consumption above recommended ${ }^{\mathrm{a}}$,

n $(\%)$

Medical history (Charlson Comorbidity Index)

CCI score 2-3 / 4-5 / 6-7 / 8+

$$
\begin{array}{ll}
21(24 \%) / 32(36 \%) / & 17(8 \%) / 106(52 \%) / \\
22(25 \%) / 13(15 \%) & 60(30 \%) / 20(10 \%)
\end{array}
$$

Location of surgery, n (\%)

Oesophagus

$31(15 \%)$

Stomach

$20(10 \%)$

Pancreas

$71(35 \%)$

Intestines

$7(3.4 \%)$

Colorectal

$64(31 \%)$

Other

$10(4.9 \%)$ 


\begin{tabular}{lccc} 
PPI-interval & $\begin{array}{c}\text { SAE } \\
(\mathbf{n}=\mathbf{1 1 7})\end{array}$ & $\begin{array}{c}\text { Mortality } \\
(\mathbf{n}=\mathbf{1 0})\end{array}$ & Unadj. OR for SAE 95\% CI \\
\hline$<\mathbf{0 . 6}(\mathbf{n}=\mathbf{3 4})$ & $21(62 \%)$ & $2(5.9 \%)$ & $3.11[1.41-6.86]$ \\
$\mathbf{0 . 6 - 1 . 4}(\mathbf{n = 1 2 9})$ & $54(42 \%)$ & $6(4.7 \%)$ & $1.38[0.82-2.33]$ \\
$\mathbf{1 . 4 - 5 . 0}(\mathbf{n}=\mathbf{1 1 4})$ & $39(34 \%)$ & $2(1.8 \%)$ & REF \\
$>\mathbf{5 . 0}(\mathbf{n}=\mathbf{1 4})$ & $3(21 \%)$ & $0(0 \%)$ & $0.52[0.14-1.99]$
\end{tabular}

PPI below 0.3

$\begin{array}{lccc}<\mathbf{2 . 5} \text { hours }(\mathbf{n = 1 9 7}) & 65(33 \%) & 6(3.0 \%) & \text { REF } \\ >=\mathbf{2 . 5} \text { hours }(\mathbf{n = 9 4}) & 52(55 \%) & 4(4.3 \%) & 2.51[1.52-4.16] \\ <\mathbf{5} \text { hours }(\mathbf{n = 2 4 9}) & 89(35.7 \%) & 8(3.2 \%) & \text { REF } \\ >=\mathbf{5} \text { hours }(\mathbf{n = 4 2}) & 28(67 \%) & 2(4.8 \%) & 3.60[1.80-7.18] \\ <\mathbf{1 0} \text { hours }(\mathbf{n = 2 7 3}) & 103(38 \%) & 8(2.9 \%) & \text { REF } \\ >=\mathbf{1 0} \text { hours }(\mathbf{n = 1 8}) & 14(78 \%) & 2(11.1 \%) & 5.78[1.85-18.02]\end{array}$



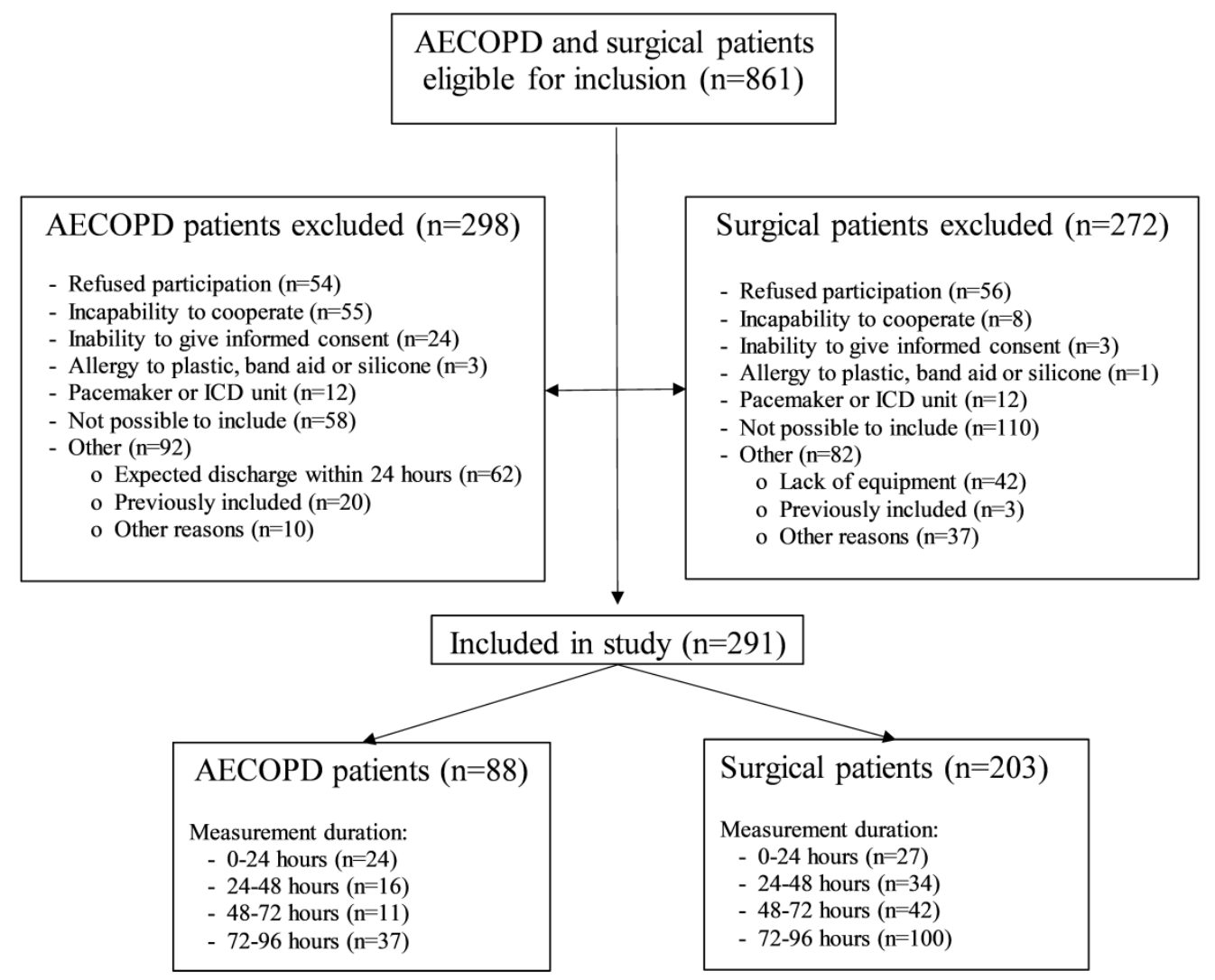

aas_13711_f1.tif 


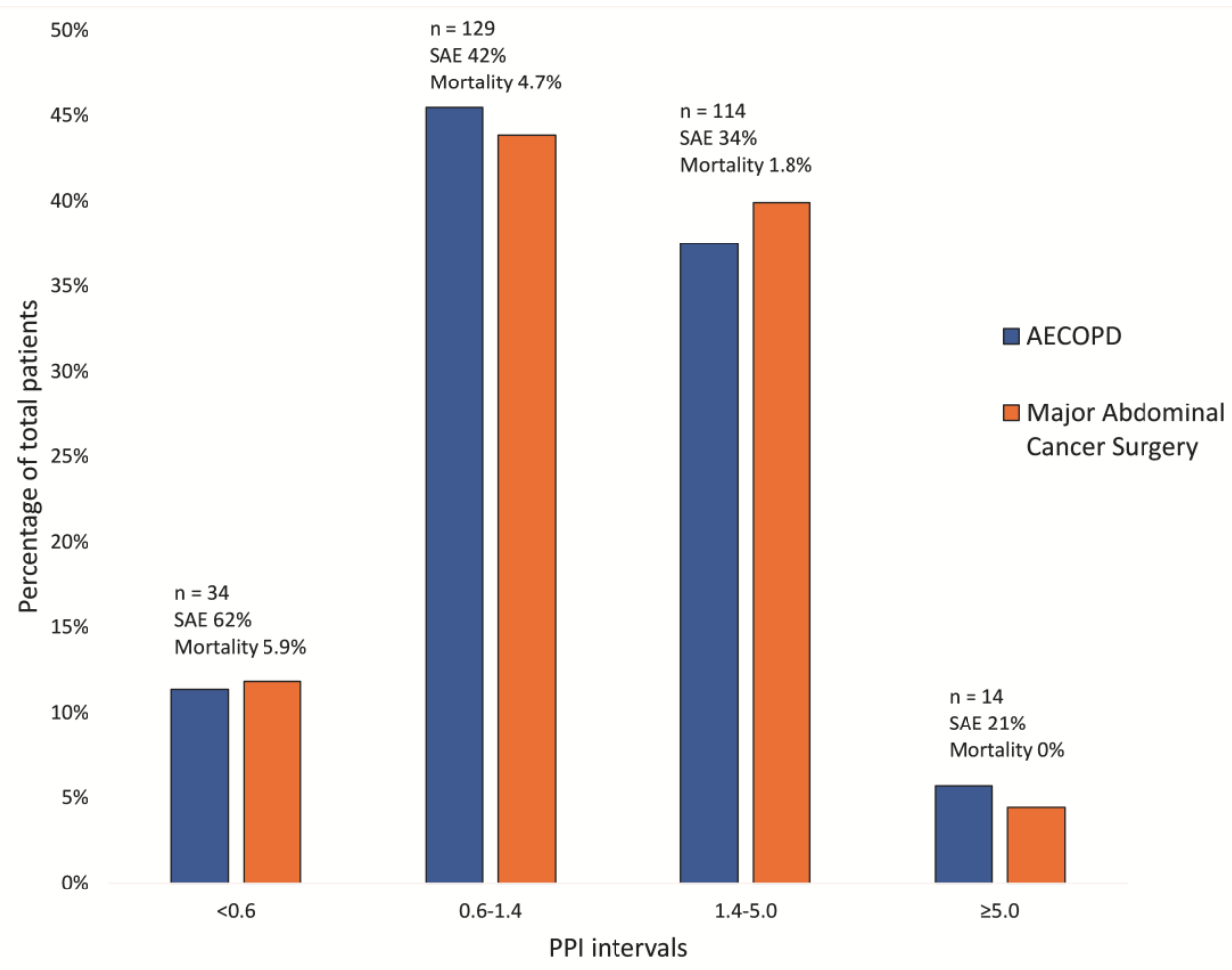

aas_13711_f2.tif 


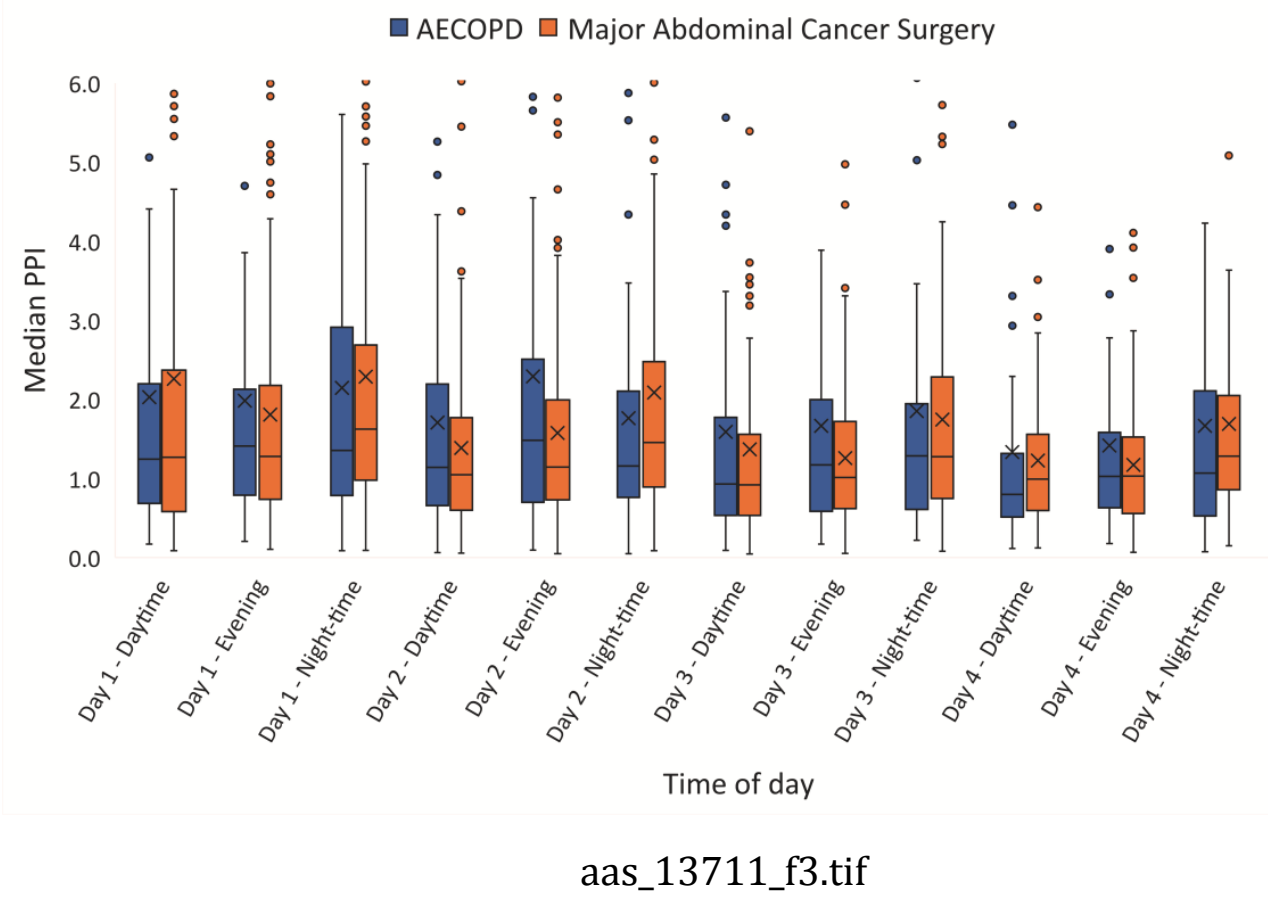

This article is protected by copyright. All rights reserved 


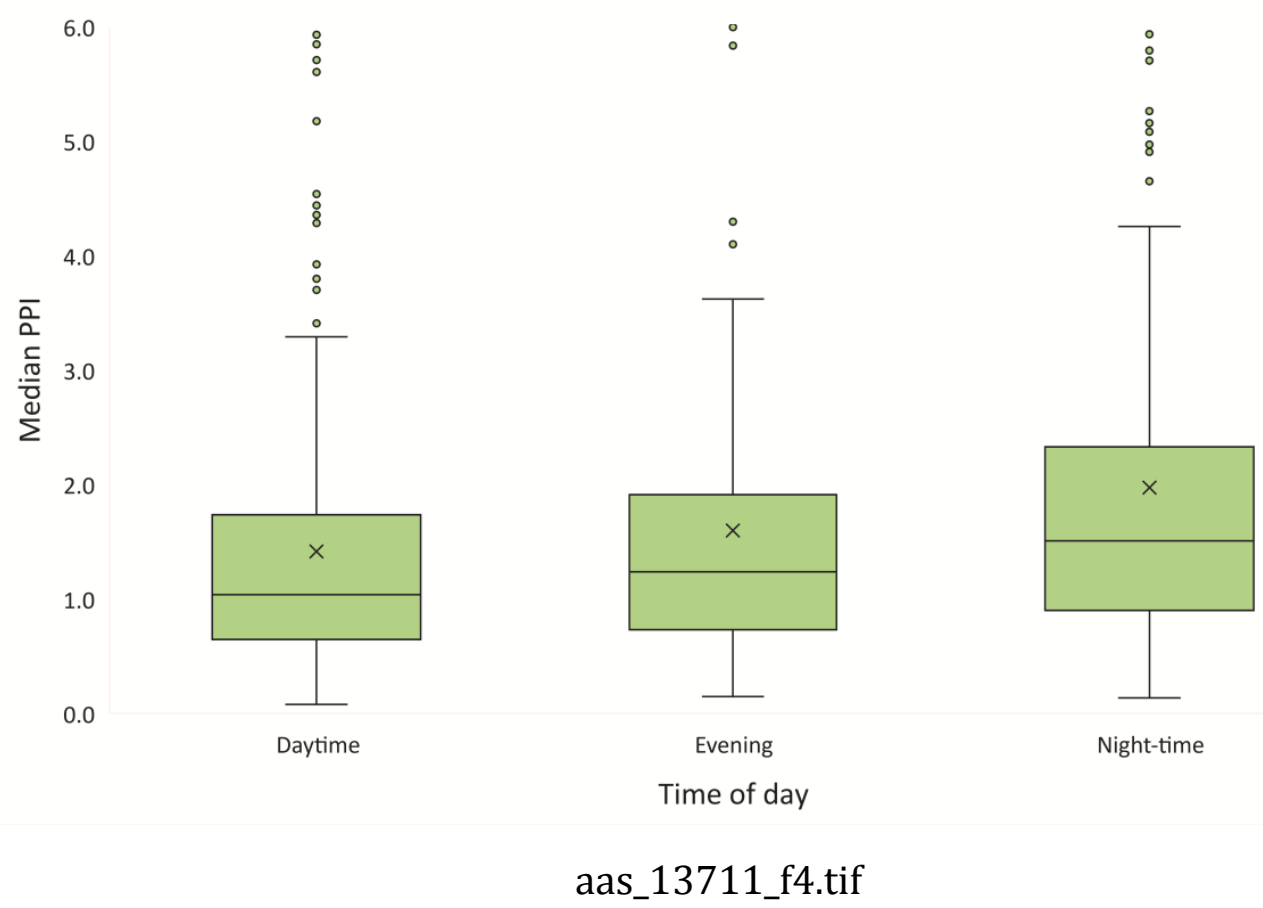

This article is protected by copyright. All rights reserved 